This item was submitted to Loughborough's Research Repository by the author.

Items in Figshare are protected by copyright, with all rights reserved, unless otherwise indicated.

\title{
Modelling the growth of ZnO thin films by PVD methods and the effects of post-annealing
}

PLEASE CITE THE PUBLISHED VERSION

http://dx.doi.org/10.1088/0953-8984/25/13/135002

PUBLISHER

(c) IOP Publishing Ltd.

VERSION

AM (Accepted Manuscript)

LICENCE

CC BY-NC-ND 4.0

\section{REPOSITORY RECORD}

Blackwell, Sabrina, Roger Smith, Steven D. Kenny, Michael Walls, and Carlos F. Sanz-Navarro. 2019. "Modelling the Growth of Zno Thin Films by PVD Methods and the Effects of Post-annealing". figshare. https://hdl.handle.net/2134/12070. 
This item was submitted to Loughborough's Institutional Repository (https://dspace.lboro.ac.uk/) by the author and is made available under the following Creative Commons Licence conditions.

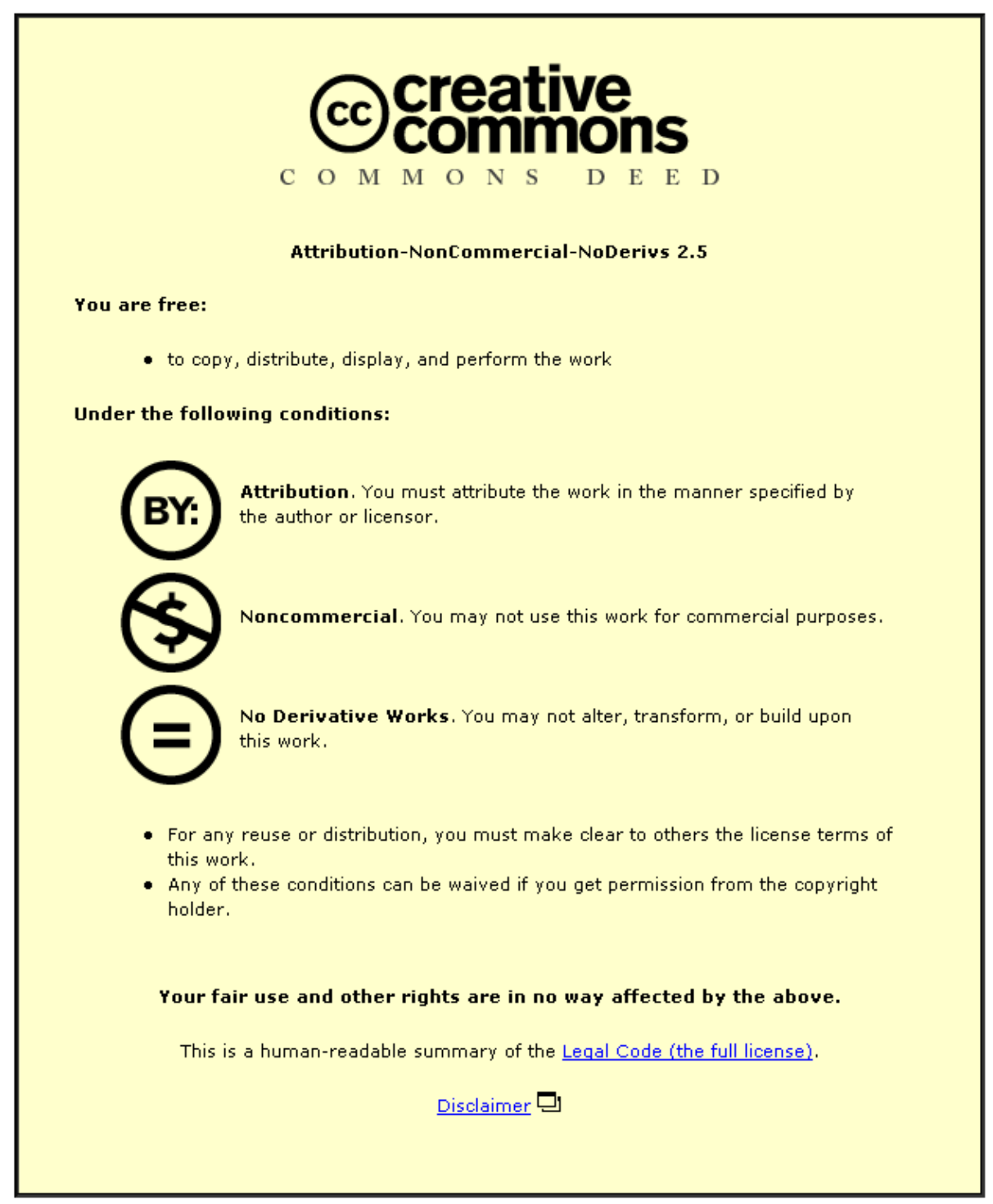

For the full text of this licence, please go to: http://creativecommons.org/licenses/by-nc-nd/2.5/ 


\title{
Modelling the growth of $\mathrm{ZnO}$ thin films by PVD methods and the effects of post-annealing
}

\author{
Sabrina Blackwell ${ }^{1}$, Roger Smith ${ }^{1}$, Steven D Kenny ${ }^{1}$, John M Walls ${ }^{2}$ and \\ Carlos F Sanz-Navarro ${ }^{3}$ \\ ${ }^{1}$ Department of Mathematical Sciences and ${ }^{2}$ School of Electronic, Electrical and Systems \\ Engineering, Loughborough University, Loughborough, Leicestershire, LE11 3TU, UK \\ ${ }^{3}$ Centre dInvestigaci en Nanocincia i Nanotecnologia (CIN2) CSIC-ICN, Campus UAB, \\ 08193 Bellaterra, Spain
}

E-mail: Sab.Blackwell@gmail.com

\begin{abstract}
Results are presented for modelling the evaporation and magnetron sputter deposition of $\mathrm{Zn}_{x} \mathrm{O}_{y}$ onto an O-terminated $\mathrm{ZnO}(000 \overline{1})$ wurtzite surface. Growth was simulated through a combination of molecular dynamics (MD) and an on-the-fly Kinetic Monte Carlo (otf-KMC) method, which finds diffusion pathways and barriers without prior knowledge of transitions. We examine the effects of varying experimental parameters, such as substrate bias, distribution of the deposition species and annealing temperature. It was found when comparing evaporation and sputtering growth, that the latter process results in a denser and more crystalline structure, due to the higher deposition energy of the arriving species. The evaporation growth also exhibits more stacking faults than the sputtered growth. Post-annealing at $770 \mathrm{~K}$ did not allow complete recrystallisation, resulting in films which still had stacking faults where monolayers formed in the zinc blende phase, whereas annealing at $920 \mathrm{~K}$ enabled the complete recrystallisation of some films to the wurtzite structure. At the latter temperature atoms could also sometimes be locked in the zinc blende phase after annealing. When full recrystallisation did not take place, both wurtzite and zinc blende phases were seen in the same layer, resulting in a phase boundary. Investigating the various distributions of deposition species found that, during evaporation, the best quality film resulted from the a stoichiometric distribution where only $\mathrm{ZnO}$ clusters were deposited. During sputtering, however, the best quality film resulted from a slightly $\mathrm{O}$ rich distribution. Two stoichiometric distributions, one involving mainly $\mathrm{ZnO}$ clusters and the other involving mainly single species, showed that the distribution of deposition species makes a huge impact on the grown film. The deposition of predominantly single species causes many more $\mathrm{O}$ atoms at the surface to be sputtered or reflected, resulting in an O deficiency of up to $18 \%$ in the deposited film and therefore resulting in more stacking faults and phase boundaries. The methods used allow analysis of key mechanisms that occur during the growth process and give hints as to the optimum conditions under which complete, crystalline layers can form.
\end{abstract}

PACS numbers: $81.15 .-\mathrm{z}$ 


\section{Introduction}

Zinc oxide is an inorganic compound with many uses, including as an additive in plastics, ceramics, glass, paints, pigments and foods. Materials science has, within the last decade, highlighted $\mathrm{ZnO}$ as an excellent alternative to other, more expensive and less abundant materials. As a transparent semiconductor, with a wide band gap of $3.37 \mathrm{eV} \mathrm{[1],} \mathrm{ZnO}$ has become the material of choice in the production of transparent electrodes for silicon thin film solar cells. $\mathrm{ZnO}$ is commonly doped with $\mathrm{Al}$ to form $\mathrm{AZO}$, a transparent conductive oxide (TCO), which is a huge industry alone within the photovoltaic sector [2]. AZO typically incorporates $0.5 \%$ of $\mathrm{Al}$, but for simplicity in the simulations, only intrinsic $\mathrm{ZnO}$ is investigated. $\mathrm{ZnO}$ also has applications in intrinsic n-type semiconductors.

Metal-oxide thin films can be deposited using a variety of industrial-scale processes, including evaporation (thermal and electron beam) and reactive magnetron sputtering [3-6]. The evaporation process involves evaporation of $\mathrm{Zn}_{x} \mathrm{O}_{y}$ molecules onto the substrate with kinetic energy typically $<1 \mathrm{eV}$. An ion source (usually argon) may also be used to densify the film by introducing energy into the growing film.

Magnetron sputtering deposits thin films of $\mathrm{ZnO}$ using RF, DC or pulsed DC power [5, 6]. Targets can be metal Zn (DC) or ZnO (RF and pulsed DC). Sputtering in the presence of a reactive gas is necessary to different degrees depending on the precise deposition configuration. For the sake of simplicity we assume that all particles arrive at the surface with an energy of $\sim 40 \mathrm{eV}$ influenced by the applied or self bias on the substrate. This is a parameter that can be varied in the model. In all cases argon is used as the working gas to sputter material from the target. Argon ion bombardment during deposition also affects the density and stoichiometry of the thin film [7], however we do not simulate the presence of argon in this simulation as studies on other oxides, namely $\mathrm{TiO}_{2}$, show that the addition of a working gas does increase crystallinity but not significantly [8] and the inclusion of argon results in a large increase in computing time.

Numerous groups have modelled deposition processes of thin films [8-12], but here we look specifically at $\mathrm{ZnO}$. In this paper we investigate, at the atomistic level, the effect of using different deposition processes on the growth of $\mathrm{ZnO}$. We focus on the difference between growth produced by the two deposition processes, the lower energy depositions $(\sim 1 \mathrm{eV})$ from evaporation and the higher energy depositions $(\sim 40 \mathrm{eV})$ used in magnetron sputtering.

Of interest also is the effect of an annealing treatment on the quality of the film. Previous experiments have studied the effect of annealing AZO films at temperatures of up to $920 \mathrm{~K}$ $\left(\sim 650^{\circ} \mathrm{C}\right)$ in order to recrystallise and reduce defects within the film, thus improving film properties [13-16].

$\mathrm{ZnO}$ crystallises in one of two main polymorphs, hexagonal wurtzite or cubic zinc blende, 
both of which are tetrahedrally coordinated. At high pressures, $\mathrm{ZnO}$ may also recrystallise into the rocksalt phase [17]. The wurtzite structure is the most stable and therefore most common form, thus it is this structure that is investigated. $\mathrm{ZnO}$, in the wurtzite hexagonal crystal structure, has alternating planes of tetrahedrally coordinated $\mathrm{O}^{2-}$ and $\mathrm{Zn}^{2+}$ stacked along the c-axis [1]. Two polar surfaces exist in the $\mathrm{ZnO}$ wurtzite phase (Zn-terminated (0001) and O-terminated $(000 \overline{1})$ ) [18], during this work we use the O-terminated surface as the initial substrate, upon which the deposition takes place.

It is known that $\mathrm{ZnO}$ can grow with inherent stacking faults in the film, often referred to as planar defects, resulting in twin boundaries $[19,20]$. In highly mismatched wurtzite substrates, there is usually some zinc blende phase separated by crystallographic defects from the wurtzite phase [20,21]. $\mathrm{ZnO}$, in thin film or nanostructure form, often contains stacking faults where both wurtzite and zinc blende phases are observed. Other materials with similar crystal structures, such as CdTe, exhibit similar stacking faults and twin boundaries where the wurtzite and zinc blende phases form side by side [22,23]. Planar defects can significantly affect the electronic properties of nanowires [24]. The difference between the zinc blende and wurtzite structures is the bond angle of the second nearest neighbours which causes a different stacking sequence of close packed planes. The wurtzite structure is made of triangularly arranged alternating close packed planes, with a stacking sequence of AaBbAaBb... in the (0001) direction [21] (later illustrated in figures 1 and 14). The zinc blende structure however along the [111] direction exhibits a $60^{\circ}$ rotation and thus consists of triangularly arranged atoms in the close packed (111) planes, causing a stacking order of AaBbCcAaBbCc... [21]. Upper and lower case letters stand for the different species. During our simulations we do indeed observe stacking faults and phase boundaries and our methods allow us to have a more precise understanding of the causes for stacking faults.

\section{Methodology}

Despite huge advances in computational power, using traditional molecular dynamics (MD) alone to model large systems over long time scales is computationally infeasible. Traditional MD allows simulation of at most a few milliseconds, depending on the system size, whereas the time required to grow one monolayer of $\mathrm{ZnO}$ experimentally is up to one second. Long time scale dynamics combines MD with new techniques, allowing simulation over experimental time scales without biasing the system dynamics [25]. In this work we apply a multi time scale technique that combines MD with on-the-fly Kinetic Monte Carlo (otf-KMC) [26]. This allows simulation of the growth of several layers.

Central to a simulation is the application of an interatomic potential function, which describes the way in which atoms will interact. For these simulations we employed a reactive force field (ReaxFF), published by van Duin in 2001 for hydrocarbons [27] and more recently for other materials including $\mathrm{ZnO}[28,29]$. Traditional force fields depend on having all bonds defined explicitly. ReaxFF, however, avoids this in favour of bond orders which allow for continuous 
bond formation and breaking, enabling a more general description of chemistry. The total energy function of a system, calculated using ReaxFF, involves a sum of all of the terms describing the individual chemical bonds in the materials, thus providing accurate simulations of chemical reactions. The substrate was modelled as a rectangular box 8 layers deep with 128 atoms per layer. Periodic boundary conditions were applied in the $\mathrm{x}$ and $\mathrm{z}$ directions. Particles were impacted normal to the surface in the y direction.

When simulating growth, we took a perfect O-terminated $\mathrm{ZnO}(000 \overline{1})$ wurtzite surface, as shown in figure 1. An ad-unit is deposited onto the surface with the desired kinetic energy by first running MD for up to $10 \mathrm{ps}$. The system is then relaxed and diffusion between deposition events is simulated using otf-KMC, described below. At each step either a deposition event or a diffusion event is chosen, according to their relative probabilities. Growth continues in this way until we have grown the number of layers desired.

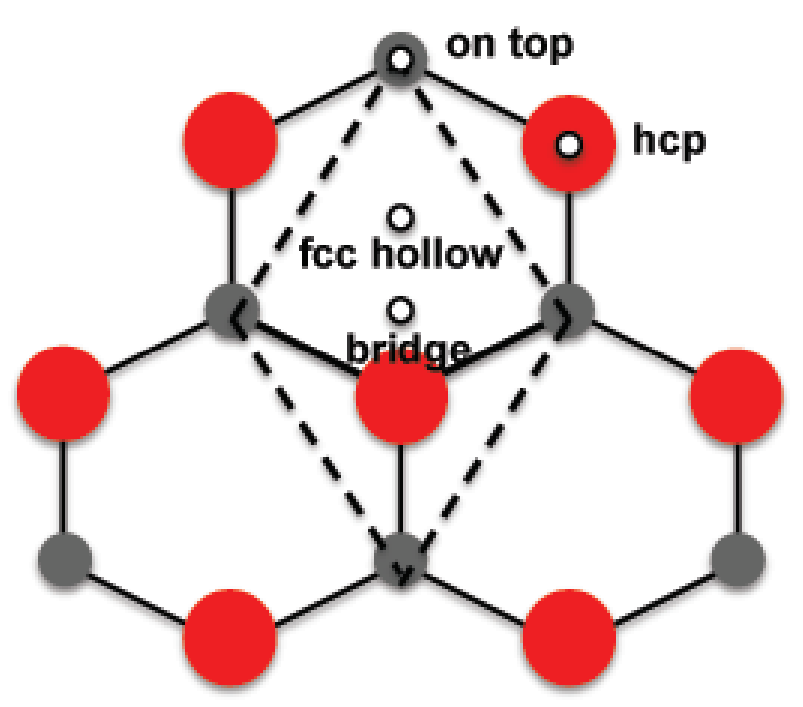

(a) O-terminated wurtzite $\mathrm{ZnO}$ surface $(000 \overline{1})$.

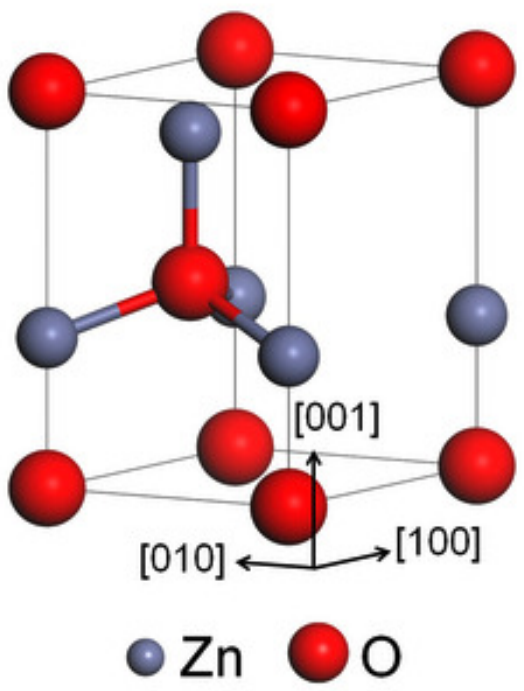

(b) Wurtzite $\mathrm{ZnO}$ unit cell [30].

Figure 1: With its hexagonal structure, $\mathrm{ZnO}$ requires arriving atoms to follow the hexagonal nature in order to continue the crystalline growth. The unit cell is shown, with the highsymmetry adsorption sites for arriving species. In order to follow the perfect crystalline growth, atoms should bind either in an on top or hcp site. Stacking faults, however, can form when atoms bind on the fcc hollow site. This is further investigated later in the section 'stacking faults'.

During the MD stage, the bottom layer of the lattice was fixed and a Berendsen [31] thermostat was attached to the next two layers to remove any excess energy from the system caused by the deposition. Randomly oriented ad-units were deposited normally to the surface, with the distribution of deposition species arriving being specific to the simulation. Deposition energies were selected from a normal distribution, with standard deviation a tenth of the de- 
position energy. The lattice was heated to $350 \mathrm{~K}$ before each deposition, and MD ran until the lattice returned to this desired temperature and defects stabilised, taking typically $4-10$ ps, depending on deposition energy used. Literature states that $\mathrm{ZnO}$ films can be deposited at temperatures ranging from room temperature $(350 \mathrm{~K})$ up to $\sim 670 \mathrm{~K}[6]$. During these simulations, the lower temperature of $350 \mathrm{~K}$ is used for the deposition temperature.

The otf-KMC algorithm involves four fundamental steps, described below:

1. Identification of all the defects by comparison to a perfect bulk lattice. This produces a search region where defects and their neighbouring atoms are included.

2. Search for all possible transitions involving only the atoms defined in the search space. Locate saddle points using the Relaxation and Translation method (RAT) [32] and then use the climbing image Nudged Elastic Band method (NEB) [33-35] to determine barrier heights more accurately once the transition has been found. Typically $\sim 200$ searches are carried out, which is enough for all unique transitions to be found.

3. Calculate transition rates of every unique transition found using the Arrhenius equation:

$$
\text { Escape Frequency }=\nu \exp \left(-E_{b} / k_{B} T\right)
$$

where $\nu$ is the transition prefactor, $E_{b}$ is transition barrier, $k_{B}$ is the Boltzmann constant and $T$ is the temperature. $\nu$ can be calculated for each transition using the Vineyard [36] method, however, due to time limitations we take the prefactor to be $10^{13}$ seconds $^{-1}$ [32]. The deposition event is also assigned a rate, which in our case is equivalent to a growth of three monolayers per second.

4. Transition searches together with a deposition event are carried out in parallel, on $48 \mathrm{cpu}$ cores, where the deposition event runs MD on a single core and searches are employed on multiple cores. Either a transition or deposition event is chosen from a roulette algorithm, and is used to evolve the system in time.

The second step of the otf-KMC method involves transition searches. Transitions will have a range of relevant energy barriers. In order to save computational time, transitions with energy barriers above $1.0 \mathrm{eV}$ were ignored. Transitions with energy barriers above $1.0 \mathrm{eV}$ would rarely occur on our time scales. During the initial test simulations of $\mathrm{ZnO}$ growth, it was found that a very low energy barrier was consuming a large amount of the simulation time, therefore slowing the simulations down unacceptably. Upon investigation these low energy barriers were found to belong to $\mathrm{O}$ dimer vibrations on the surface. An $\mathrm{O}$ dimer vibrating adds no net diffusion, hence we were able to modify the methods in order to ignore these vibrational transitions and thus save computational time.

Post-annealing the substrate is achieved by running MD at an elevated temperature. The literature states that $\mathrm{ZnO}$ films have been annealed at temperatures of up to 920K [13-16]. Once a growth simulation has completed, we can simulate a post-annealing treatment by running high temperature MD on the system. A time of $10 \mathrm{~ns}$ was found to be appropriate. The system 
is then relaxed using conjugate gradient minimisation. The systems, pre- and post- annealing, were then compared.

\section{Results}

In this section results are presented for growth via two deposition processes, evaporation and reactive magnetron sputtering. An advantage of these methods is the ability easily to change parameters. An important parameter is the distribution of various deposition species arriving at the surface and it is not fully understood how variations of precise distribution affect the resulting film composition. Various distributions of deposition species have been studied, including $\mathrm{O}$ rich, $\mathrm{O}$ deficient and stoichiometric. Table 1 describes the exact distribution of deposition species used during the simulations. Within the stoichiometric distributions, for evaporation it is assumed that the majority of species arriving at the substrate would be $\mathrm{ZnO}$ units. Whereas for sputtering, two variations of distribution are considered. Sputtering A assumes that most $\mathrm{Zn}$ atoms oxidise in the plasma en route to the substrate, whilst sputtering $\mathrm{B}$ assumes no reactive gas is used and therefore a large part of arriving species are single $\mathrm{Zn}$ and $\mathrm{O}$ and $\mathrm{O}_{2}$ atoms rather than $\mathrm{ZnO}$ clusters.

\begin{tabular}{cccccc}
\hline Species & $\begin{array}{c}\text { Stoichiometric } \\
\text { (evaporation) }\end{array}$ & $\begin{array}{c}\text { Stoichiometric } \\
\text { (sputtering A) }\end{array}$ & $\begin{array}{c}\text { Stoichiometric } \\
\text { (sputtering B) }\end{array}$ & O rich & O deficient \\
\hline $\mathrm{O}$ & 0.0 & 0.0 & 0.1 & 0.02 & 0.0 \\
$\mathrm{O}_{2}$ & 0.0 & 0.04 & 0.2 & 0.15 & 0.0 \\
$\mathrm{Zn}$ & 0.0 & 0.1 & 0.5 & 0.08 & 0.1 \\
$\mathrm{ZnO}$ & 1.0 & 0.86 & 0.2 & 0.75 & 0.9 \\
\hline
\end{tabular}

Table 1: The distribution of various deposition species arriving at the surface at normal incidence.

\section{Evaporation Deposition}

Deposition using evaporation is an industrial process used to grow $\mathrm{ZnO}$ films [3,4], modelled here by $1 \mathrm{eV}$ normal deposition. After simulating each of the flux distributions, we found that the best quality, evaporated film resulted from the simulation where all arriving species were $\mathrm{ZnO}$ clusters. Figure 2 shows the resulting film, and it is clear that the original substrate is undamaged and still perfectly crystalline, whilst the first new layer down is stoichiometric and almost complete. Atoms in the second new layer are not all in the expected stacking configuration and crystalline sites, resulting in a stacking fault. The layer is slightly $\mathrm{O}$ rich. The low arrival energy of the $\mathrm{ZnO}$ molecules means that atoms will not all have enough energy to pass activation barriers and diffuse into correct lattice sites. The equivalent of three monolayers of atoms are deposited during the simulation but there are actually four new layers, illustrating that the deposited structure is not dense or complete. 

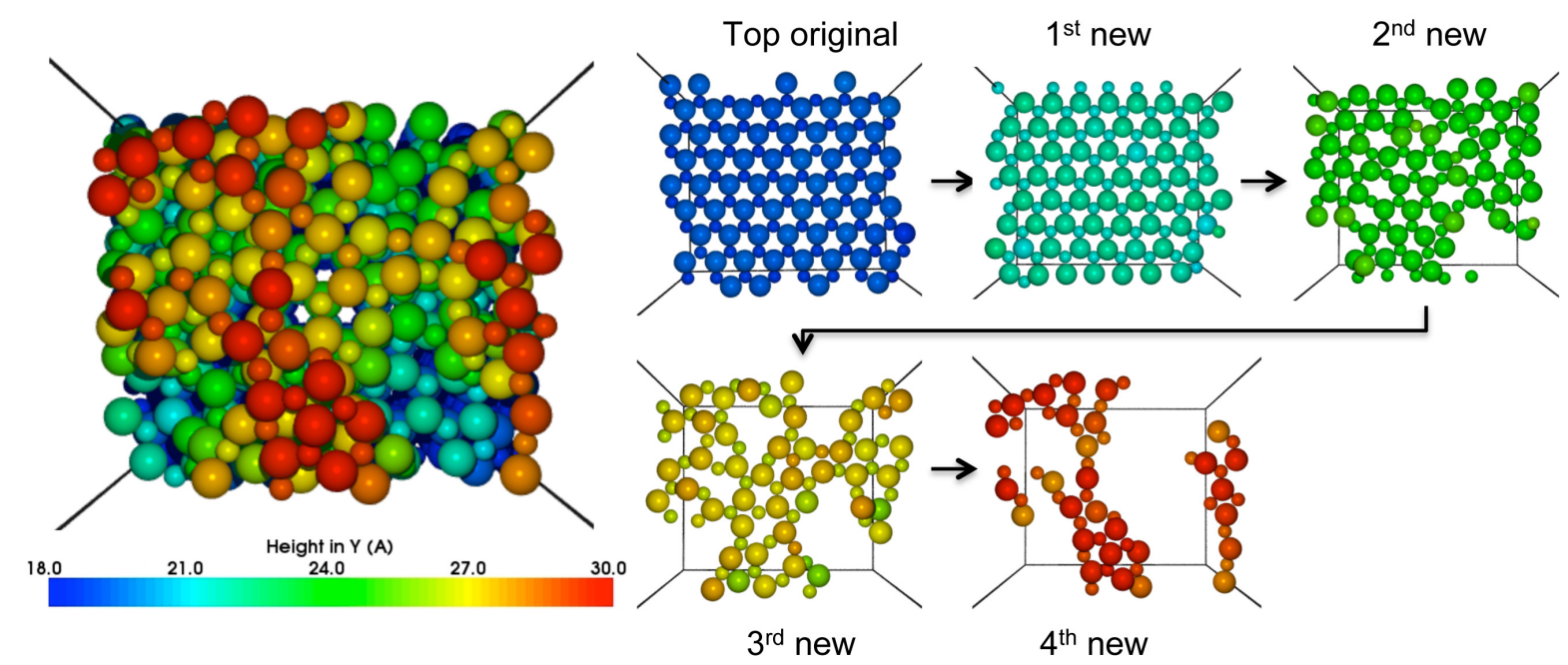

Figure 2: The evaporation deposition grown $\mathrm{ZnO}$ film, where a stoichiometric distribution of species was deposited. Larger spheres represent $\mathrm{O}$ and smaller spheres represent $\mathrm{Zn}$. Atoms are coloured by height as shown on the colour bar. Layers have been broken down for clarity purposes, with the original surface at top left, followed by first new layer and so on, ending with the last new layer. The second new layer shows a stacking fault where atoms are not in correct sites.

Post-annealing of $\mathrm{ZnO}$ films at $920 \mathrm{~K}$, has the ability to recrystallise the structure and decrease the number of defects [13-16]. Using MD we have simulated the annealing process. Figures 3 and 4 show the effects of annealing the system shown in figure 2 at $770 \mathrm{~K}$ and at $920 \mathrm{~K}$ respectively. At $770 \mathrm{~K}$ there is some recrystallisation, however due to an inherent stacking fault already present, a phase boundary forms in the film, where wurtzite and zinc blende are both present and this is discussed later. At $920 \mathrm{~K}$ there is almost complete recrystallisation and all stacking faults are eradicated.

$\mathrm{O}$ deficient and $\mathrm{O}$ rich distributions were also simulated, producing less complete films than the stoichiometric distribution, where layers are incomplete and show low density with stacking faults throughout the new layers. Post-annealing at $920 \mathrm{~K}$ transfers sufficient energy to the lattice for atoms to drop down, completing layers below and thus densifying the film. However, all new layers become stuck in the zinc blende phase, suggesting that $\mathrm{O}$ deficiency promotes the presence of stacking faults.

\section{Sputter Deposition}

The best quality growth, shown in figure 5, resulted from sputtering an $\mathrm{O}$ rich distribution of species, as defined in table 1 . We observe a higher density of film, as the fourth layer here has very few atoms. The original substrate surface and first new layer are undamaged and complete. The second new layer does have some defects, however, and is slightly $\mathrm{O}$ deficient. 

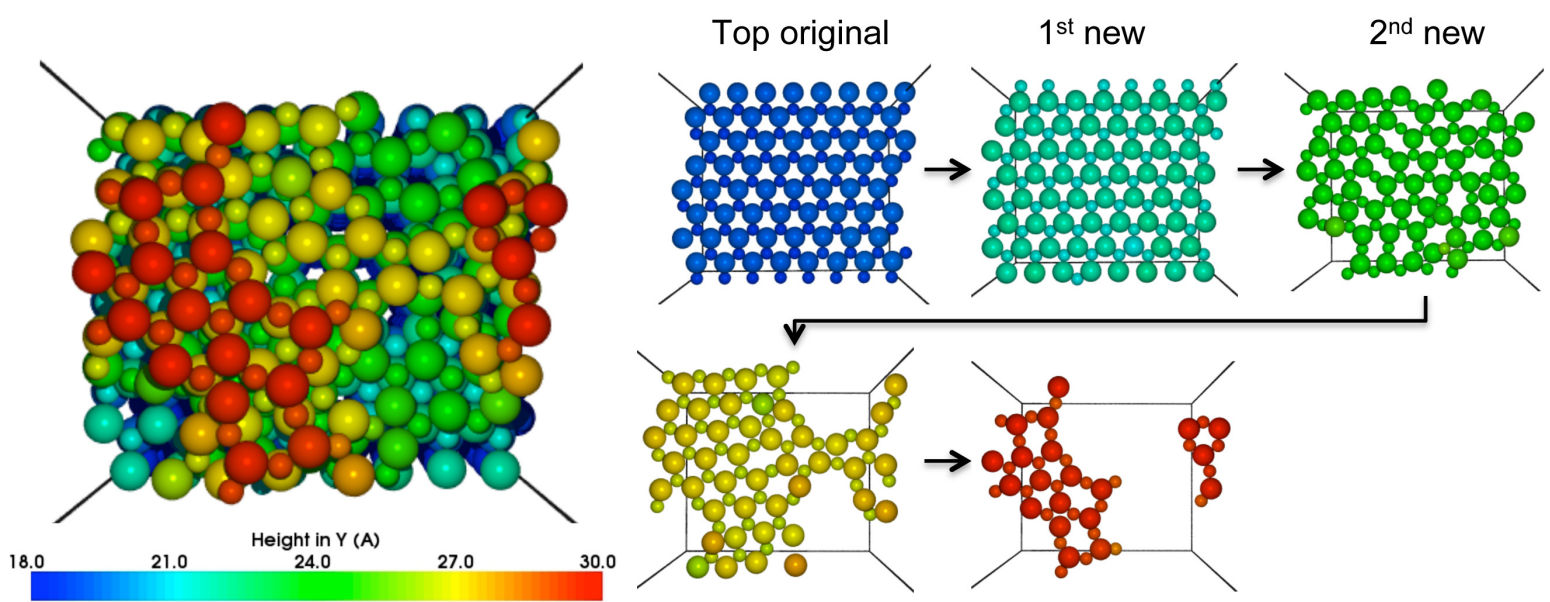

$3^{\text {rd }}$ new

$4^{\text {th }}$ new

Figure 3: The post-annealed evaporation grown film where a stoichiometric distribution of species was deposited. The system was heated to $770 \mathrm{~K}$ for $10 \mathrm{~ns}$ to simulate the postannealing treatment. Results indicate that the temperature is not high enough for complete recrystallisation to take place. The first new layer is complete, the post-annealing process has removed a point defect which was previously present. The second new layer, however, has failed to recrystallise into the correct sites and a stacking fault previously apparent has formed a phase boundary due to the annealing process allowing the layer to become $\mathrm{O}$ deficient (this is discussed later).
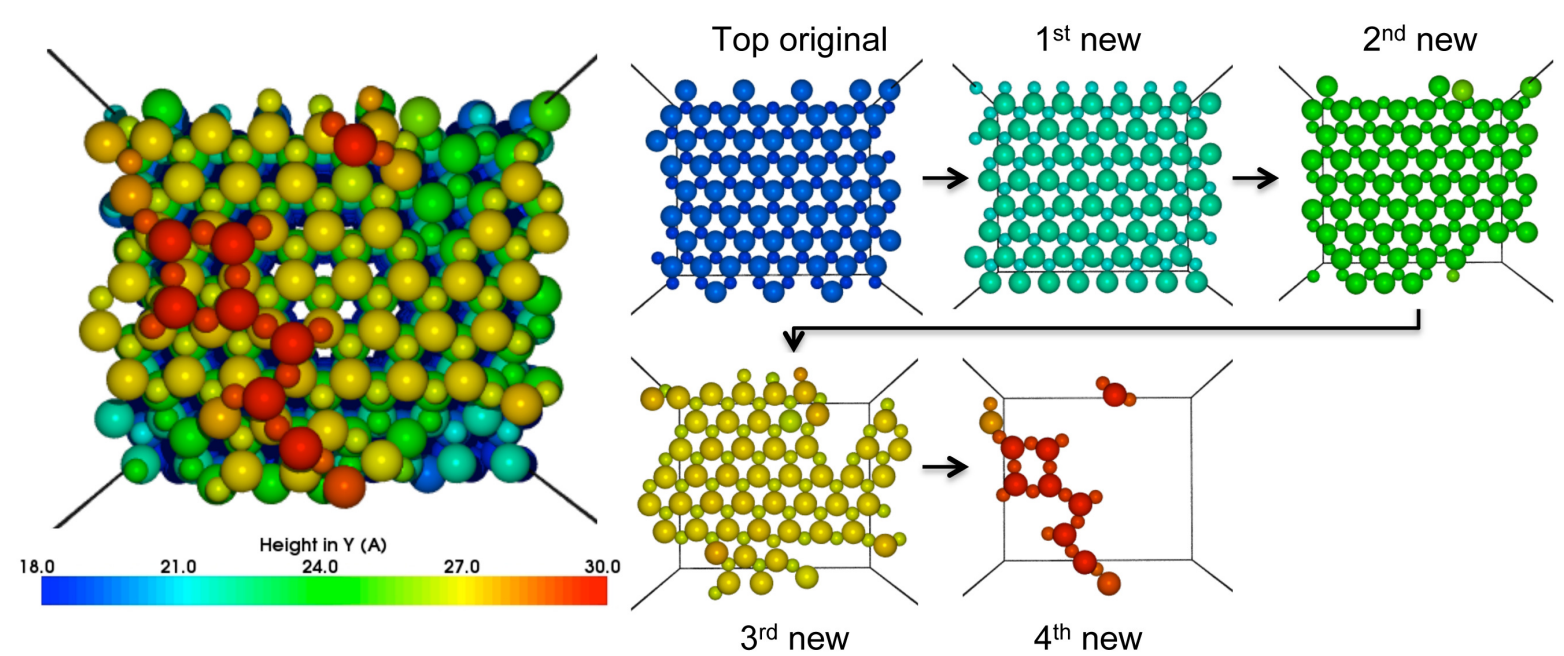

Figure 4: The post-annealed evaporation grown film where a stoichiometric distribution of species was deposited. The system was heated this time to $920 \mathrm{~K}$ to simulate a more aggressive post-annealing treatment. In this case perfect, crystalline $\mathrm{ZnO}$ has formed. The first, second and third new layers are all in the wurtzite phase with no stacking faults or phase boundaries present. 


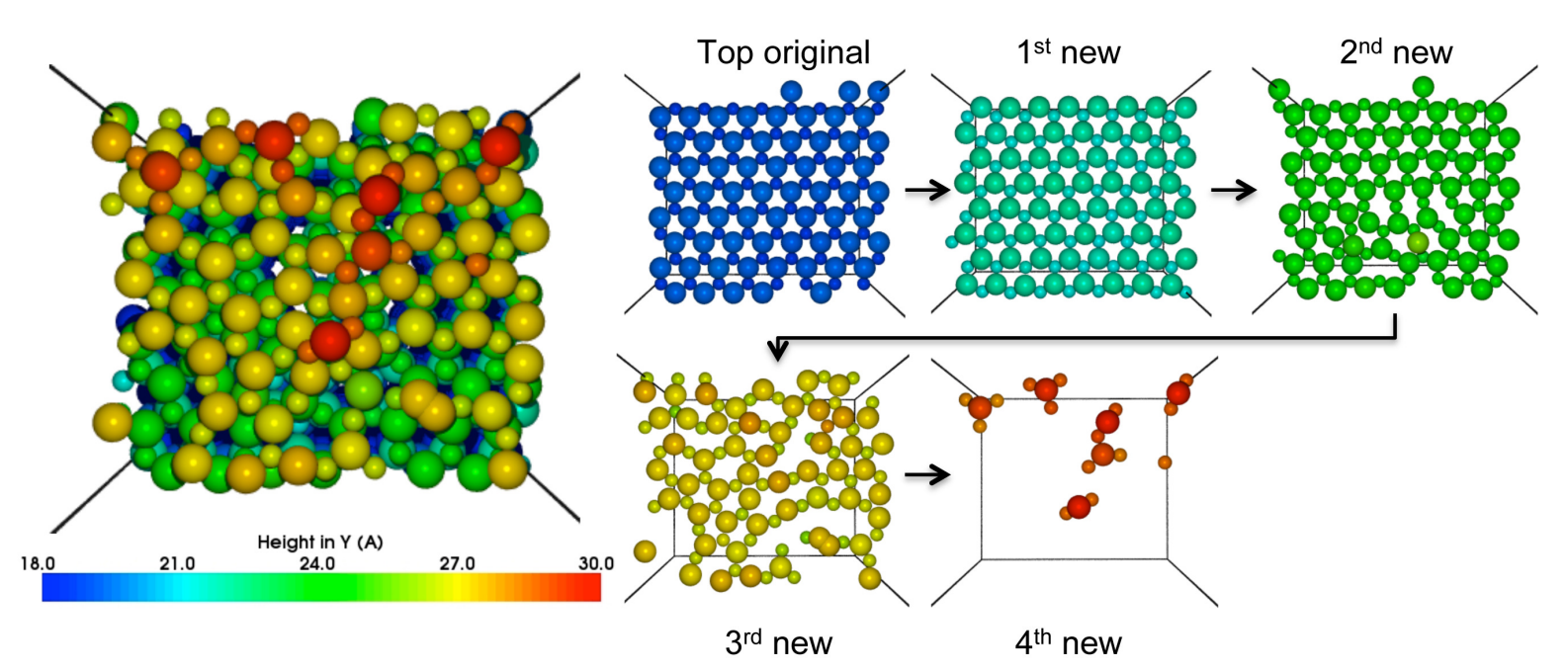

Figure 5: The resulting film from the sputter deposition of an $\mathrm{O}$ rich distribution of species, after the deposition of the equivalent to three new layers. Due to new layers not being totally complete we have four new layers. However, with only 21 atoms in the fourth layer, we would expect these to complete the layer below if growth continued and reached a steady state. The first new layer is stoichiometric and complete, whilst the second and third new layers are very slightly $\mathrm{O}$ deficient and not all atoms are sitting in correct lattice sites.

Sputtering alone cannot enable the complete and crystalline growth which is often sought, for example in photovoltaic uses. The increased deposition energy does allow increased diffusion of atoms into correct sites, however, layers are still incomplete and contain phase boundaries. Figure 6 shows, in comparison, the film after an annealing treatment, heating the system to 920K for $10 \mathrm{~ns}$. Comparing pre-annealed and post-annealed structures it is clear that the extra temperature has caused the movement of atoms into correct sites. The film still has an $\mathrm{O}$ deficiency but the completed layers are perfectly crystalline.

Stoichiometric distributions of deposition species did also provide good results of film growth. As described in table 1, two distributions were considered for the sputtering of stoichiometric $\mathrm{ZnO}$, sputtering A and sputtering B. The first stoichiometric distribution, sputtering A, assumes that the majority of species arrive at the substrate as $\mathrm{ZnO}$ units. The growth resulting from this distribution is shown in figure 7. It is clear that there is an $\mathrm{O}$ deficiency throughout the newly deposited film of $10 \%$, although the original surface and the first new layer are almost perfect with only one point defect from a missing $\mathrm{O}$ atom. The second new layer does have higher $\mathrm{O}$ deficiency compared to the first new layer, with stacking faults and $\mathrm{O}$ vacancies. The second stoichiometric distribution considered, sputtering B, assumes that $\mathrm{Zn}$ and $\mathrm{O}$ atoms rarely combine in the plasma, arriving often as single $\mathrm{Zn}$ or O species onto the substrate. We were able to investigate the effect of this variation in the species distribution by comparing the subsequent growth from the sputtering B distribution, shown in figure 8 . The original 


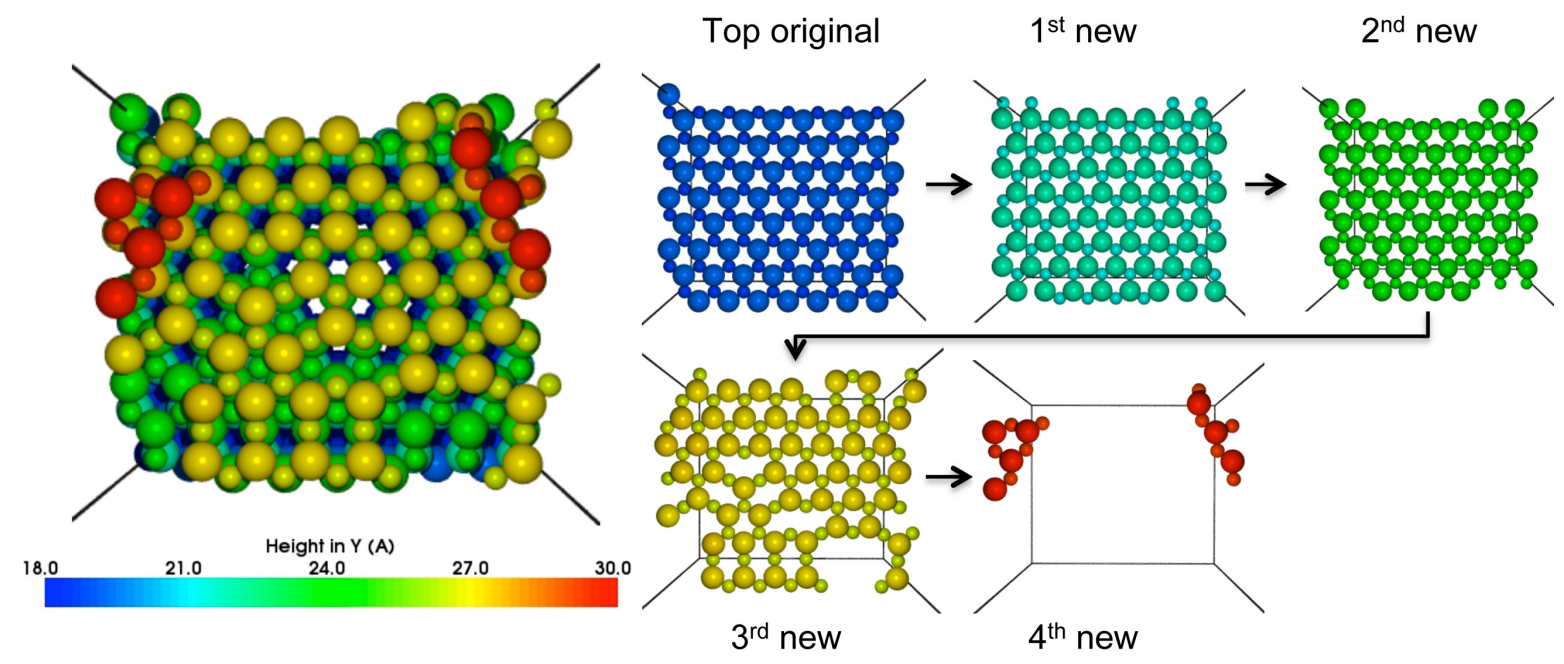

Figure 6: The post-annealed, sputter deposited film, where an O rich distribution of deposition species was used. The post-annealing treatment involved heating the system to $920 \mathrm{~K}$ for 10 ns. The first two new layers are now perfectly crystalline and in the wurtzite phase. The third new layer is $\mathrm{O}$ deficient and a stacking fault with a phase boundary has formed between the wurtzite and zinc blende phases.

substrate has an $\mathrm{O}$ vacancy, which causes some point defects. The first new layer exhibits a large $\mathrm{O}$ deficiency, along with a highly mismatched phase structure. The high defect density, coupled with the $\mathrm{O}$ deficiency has allowed the formation of a phase boundary between the wurtzite and zinc blende phases, described more fully later. The second new layer, is again $\mathrm{O}$ deficient and highly mismatched, with many defects. The $\mathrm{O}$ deficiency throughout the newly deposited film for this distribution is almost double that of the sputtering A configuration at $18 \%$.

It is now clear that the distribution of deposition species plays a huge role on the resulting growth. The distribution described by sputtering A, where largely $\mathrm{ZnO}$ units and a very small amount of single $\mathrm{Zn}$ and $\mathrm{O}_{2}$ dimers were deposited, has produced a slightly $\mathrm{O}$ deficient film with a first new layer which is almost perfectly ordered in the correct wurtzite phase. Conversely, sputtering B, where over half of arriving species were single atoms, has produced a much more $\mathrm{O}$ deficient film with unordered and mismatched layers where stacking faults exist and phase boundaries are already evident. Even post-annealing at 920K, as shown in figure 9, does not allow atoms to move into correct lattice sites. The phase boundary in the first new layer has become frozen in due to the $\mathrm{O}$ deficiency. $\mathrm{O}$ atoms have been drawn down from the surface in an attempt to restore the stoichiometry of layers below, however, this addition of $\mathrm{O}$ atoms is not enough to eradicate any faults or phase boundaries.

An interesting comparison between the simulations of the two sputtering deposition distributions ( $\mathrm{A}$ and $\mathrm{B}$ ) is the behaviour of deposition species at the surface. The first simulation, 

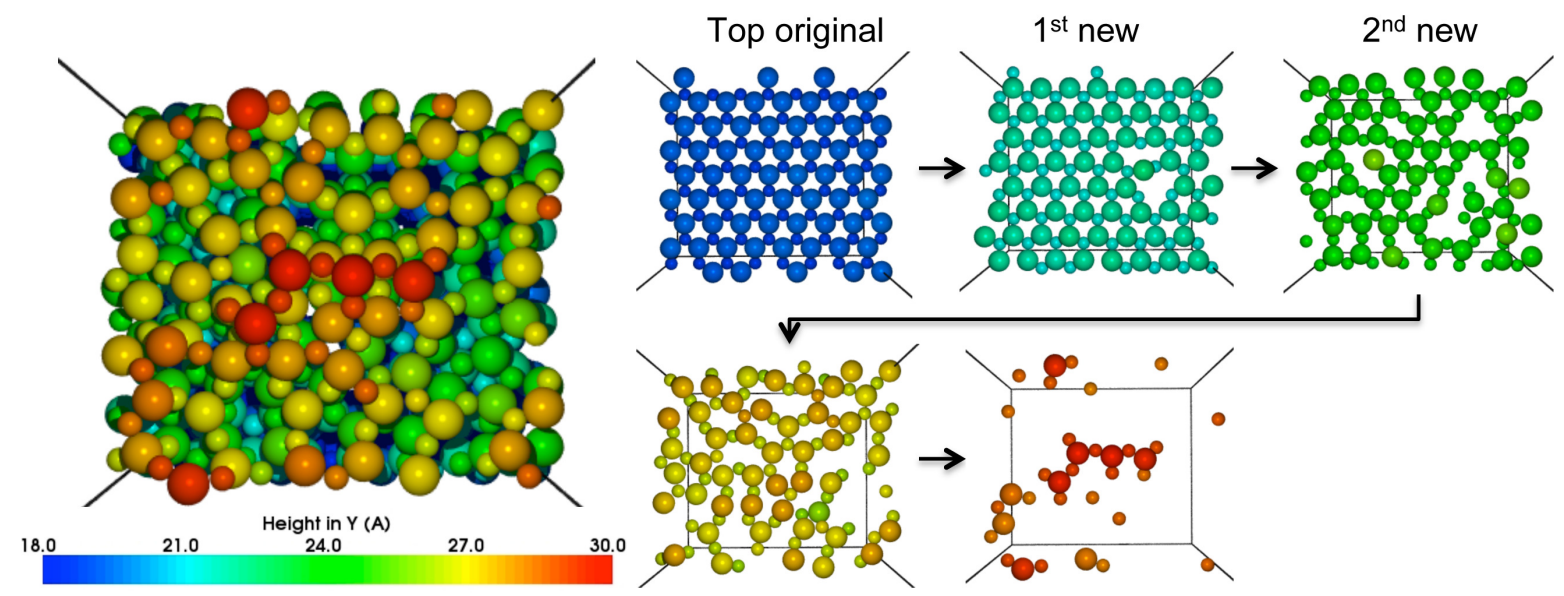

$3^{\text {rd }}$ new

$$
4^{\text {th }} \text { new }
$$

Figure 7: A sputter deposited film where the distribution of deposition species was a stoichiometric mix where most species arrived at the surface as $\mathrm{ZnO}$ units (sputtering $\mathrm{A}$ distribution described in table 1). O deficiency is evident throughout the deposited film (10\% $\mathrm{O}$ deficient), although the original surface and the first new layer are almost perfect with only one point defect from a missing $\mathrm{O}$ atom. The second new layer does have higher $\mathrm{O}$ deficiency, with stacking faults and $\mathrm{O}$ vacancies.

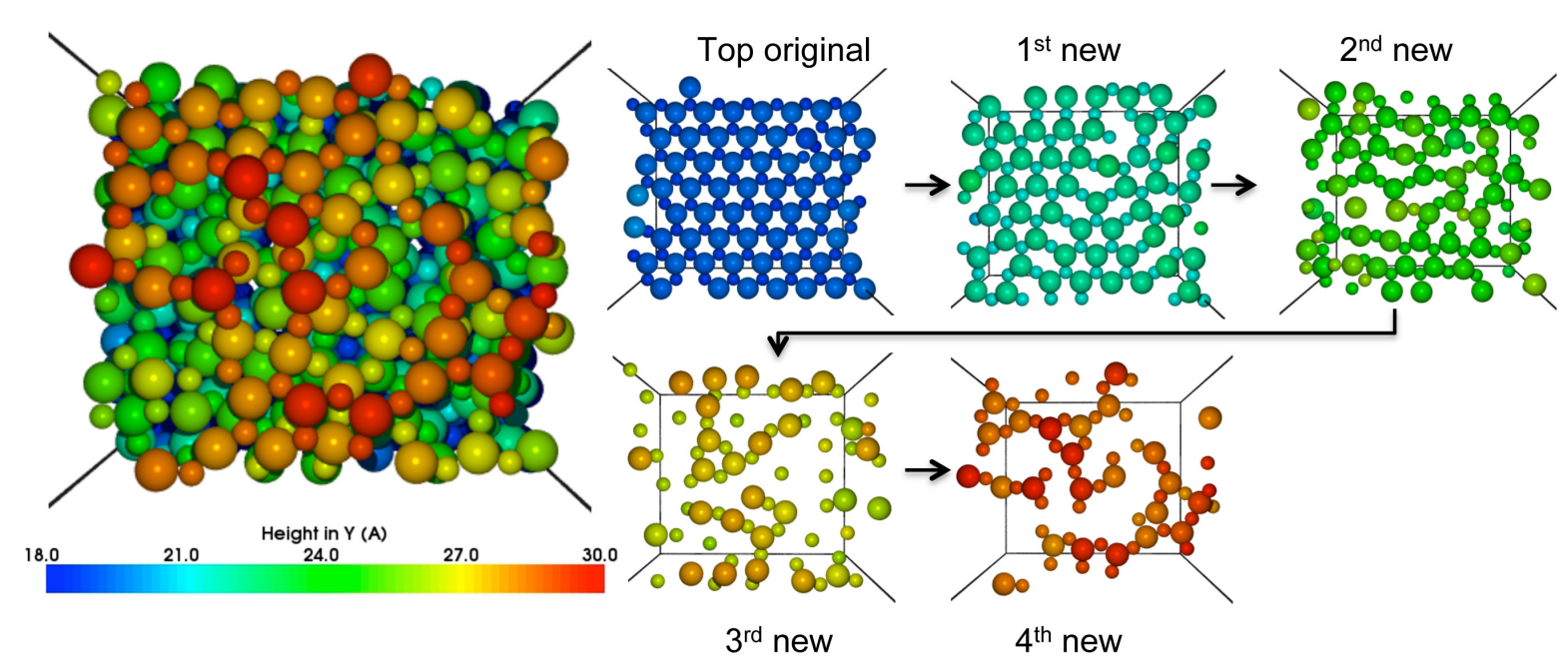

Figure 8: A sputter deposited film where the distribution of deposition species was a stoichiometric mix where most species arrived at the surface as single $\mathrm{Zn}$ or $\mathrm{O}$ species (sputtering $\mathrm{B}$ distribution described in table 1), resulting in high $\mathrm{O}$ deficiency $(18 \% \mathrm{O}$ deficient). The original substrate exhibits an $\mathrm{O}$ vacancy and the first new layer exhibits a large $\mathrm{O}$ deficiency along with a highly mismatched phase structure. The $\mathrm{O}$ deficiency and high defect density has allowed a phase boundary (highlighted) to form between the wurtzite and zinc blende phases. The second new layer is also $\mathrm{O}$ deficient and highly mismatched with stacking faults and point defects throughout. 

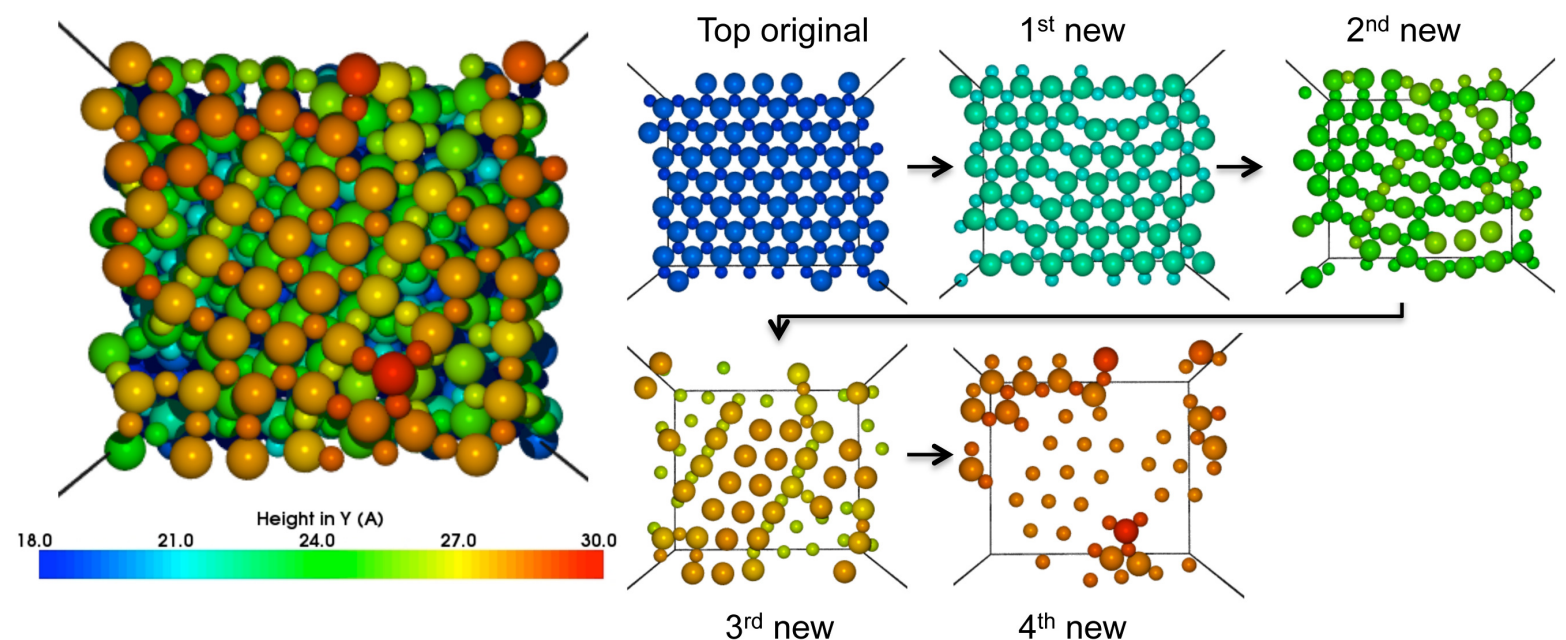

Figure 9: The post-annealed, sputter deposited film, where the stoichiometric sputtering B distribution of deposition species was used. The post-annealing treatment, heating the system to $920 \mathrm{~K}$ for $10 \mathrm{~ns}$, has not been capable here of recrystallising the atoms. Instead, stacking faults already present have become fixed, with phase boundaries forming around areas of zinc blende.

sputtering $\mathrm{A}$, observes a large proportion of surface $\mathrm{O}$ sputtered by the arrival of $\mathrm{ZnO}$ clusters. The sputtering $\mathrm{B}$ simulation, however, observes a large portion of arriving $\mathrm{O}$ and $\mathrm{O}_{2}$ either reflecting or sputtering other $\mathrm{O}$ atoms from the surface, along with a small proportion of arriving $\mathrm{Zn}$ also sputtering $\mathrm{O}$ atoms. Some $\mathrm{ZnO}$ depositions do still cause the sputtering of $\mathrm{O}$ atoms. The difference in behaviour of the species deposited thus plays a huge role in the subsequent growth quality.

During all simulations $\mathrm{O}$ and $\mathrm{O}_{2}$ reflect off the surface, are sputtered from the surface or evaporate during MD stages (around 0.1-0.2\% of depositions involve an $\mathrm{O}$ or $\mathrm{O}_{2}$ cluster reflecting off the surface). As described above, $\mathrm{O}$ ejection from the surface is caused by the deposition of any of the deposition species. This leaves an inherent $\mathrm{O}$ deficiency in the film, which reflects results seen in the literature [37]. No Zn atoms have been observed leaving the surface, agreeing with experimental evidence that films are $\mathrm{Zn}$ rich [38]. We do indeed observe an $\mathrm{O}$ deficiency in all of our simulations. The closest film to stoichiometric is the evaporated film grown using a stoichiometric distribution of deposition species where only $\mathrm{ZnO}$ is deposited ( $0.006 \% \mathrm{O}$ deficiency). The highest $\mathrm{O}$ deficiency (18\%), however, is observed from sputtered growth using a stoichiometric distribution (sputtering B), where mainly single species arrive at the surface, increasing the number of $\mathrm{O}$ atoms ejected from the substrate.

During early stages of growth, $\mathrm{Zn}$ atoms bind to the O-terminated surface. Free $\mathrm{O}$ ad-atoms or $\mathrm{O}_{2}$ dimers aid the $\mathrm{Zn}$ diffusion by binding to the $\mathrm{Zn}$, as illustrated in figure 10. This ad-atom cluster can then diffuse across the surface. The $\mathrm{Zn}$ atom then splits from the cluster when it 
reaches an appropriate site. Energies required for these transitions are typically below 0.35 $\mathrm{eV}$, with $\mathrm{ZnO}_{2}$ unit diffusion requiring as little as $0.1 \mathrm{eV}$. Although $\mathrm{Zn}$ diffusion takes place preferentially when bonded to an $\mathrm{O}$ or $\mathrm{O}_{2}$ dimer, single $\mathrm{Zn}$ atoms can also diffuse. Figure 11 illustrates an important single atom $\mathrm{Zn}$ transition whereby a $\mathrm{Zn}$ atom below the surface sitting in an interstitial site diffuses towards a surface lattice site, requiring only $0.09 \mathrm{eV}$. This mechanism aids the crystallisation of the structure during growth. Other single $\mathrm{Zn}$ atom diffusion pathways over the surface, typically involving a hop of the atom to an adjacent lattice site, require $\sim 0.2 \mathrm{eV}$. Thus, the least energy expensive method for $\mathrm{Zn}$ diffusion on the surface is by bonding to $\mathrm{O}_{2}$ dimers to form $\mathrm{ZnO}_{2}$.

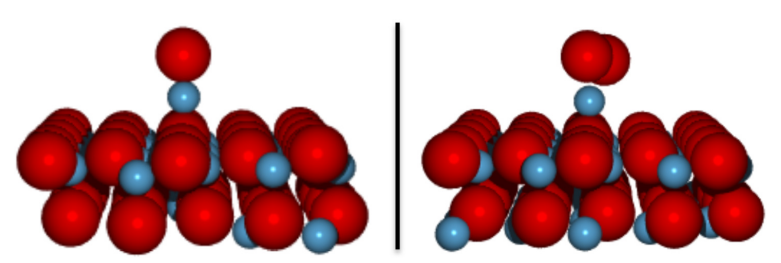

Figure 10: $\mathrm{A} \mathrm{Zn}$ atom on the surface is often bound by a $\mathrm{O}$ or $\mathrm{O}_{2}$ dimer to form a $\mathrm{ZnO}$ or $\mathrm{ZnO}_{2}$ cluster, both of which are illustrated here. The energy required for a $\mathrm{ZnO}$ ad-unit to diffuse on the surface is between $0.15 \mathrm{eV}$ and $0.35 \mathrm{eV}$, whereas a $\mathrm{ZnO}_{2}$ unit requires much less (usually $<0.1 \mathrm{eV})$. Smaller, grey spheres represent $\mathrm{Zn}$, whilst larger, red spheres represent $\mathrm{O}$.

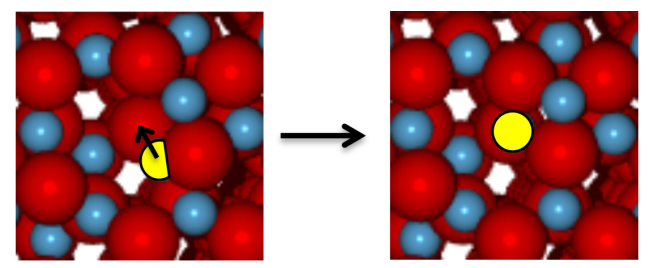

Figure 11: During a simulation, interstitials form below the surface due to bombardment and disruption of the surface. The energy required for this $\mathrm{Zn}$ interstitial, marked in yellow, to diffuse to its correct lattice site, is $0.09 \mathrm{eV}$. This small barrier indicates that this is a key mechanism by which $\mathrm{Zn}$ atoms reach their correct sites and thus crystalline $\mathrm{ZnO}$ is formed.

An important mechanism for the nucleation of $\mathrm{ZnO}$ growth is the formation and movement of $\mathrm{Zn}_{x} \mathrm{O}_{y}$ strings on the surface. These strings form on the surface via complicated, concerted motions as illustrated in figure 12, where $0.44 \mathrm{eV}$ is required for the formation of the string. Figure 13 illustrates the behaviour of these string like structures, where the vibration of a string on the surface occurs with energy barriers of between 0.2 and $0.3 \mathrm{eV}$. Later in the simulation, after tens of milliseconds, neighbouring strings and atoms become attached, forming a larger structure of strings, with a formation energy of $0.42 \mathrm{eV}$. Finally, in order for crystalline $\mathrm{ZnO}$ to form, some hundreds of milliseconds later, the strings interconnect even more and diffuse and flip to form a hexagonal structure on the surface. 
(a)

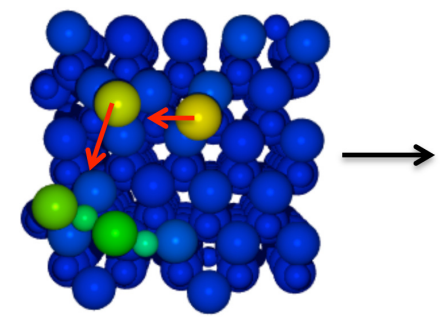

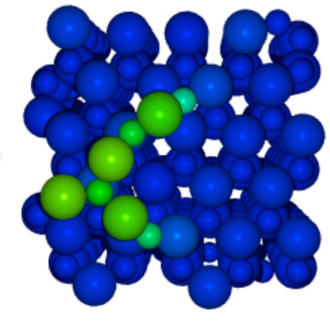

(b)

Figure 12: Two $\mathrm{ZnO}$ units marked by the arrows move as indicated towards a $\mathrm{Zn}_{2} \mathrm{O}_{2}$ string to form a longer string. The $\mathrm{Zn}$ atom lies under the $\mathrm{O}$ atom in the unit and is not visible in (a). This transition involves a concerted motion of 5 atoms, requiring $0.44 \mathrm{eV}$ to occur. Smaller spheres represent $\mathrm{Zn}$, larger spheres represent $\mathrm{O}$ and the atoms are coloured by height, where the atoms higher up are lighter in colour.
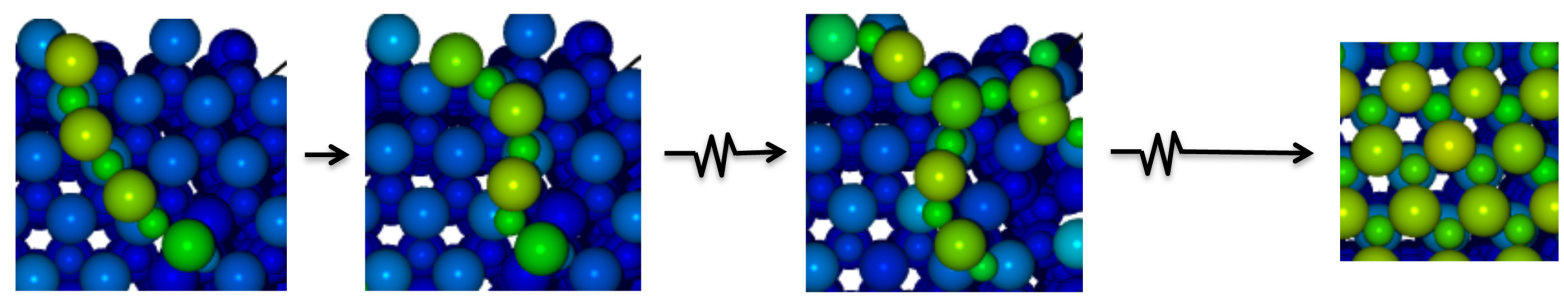

Figure 13: $\mathrm{Zn}_{x} \mathrm{O}_{y}$ strings on the $\mathrm{ZnO}$ surface act as a nucleation site for growth. These strings move with small energy barriers $(0.2-0.3 \mathrm{eV})$, as shown in the first two configurations. The third configuration shows strings forming together with new atoms joining on to make larger structures, overcoming a barrier of $0.42 \mathrm{eV}$. Finally, after hundreds of milliseconds, a hexagonal structure forms, as required for the crystalline $\mathrm{ZnO}$ growth.

\section{Stacking Faults}

Stacking faults and twin boundaries have been observed experimentally [21], however, what is not yet understood is how stacking faults occur. The correct wurtzite structure would be the $\mathrm{AaBbAaBb}$ stacking sequence, however, we observe some zinc blende (AaBbCc) structures during some of the simulations. Figure 14 shows the difference between the two phases of $\mathrm{ZnO}$. The most obvious difference is the filling of fcc hollow sites in the zinc blende phase (see figure 1 for high symmetry adsorption sites). The hcp site used in the wurtzite phase is the most stable site for an $\mathrm{O}$ atom. However, the energy difference between an $\mathrm{O}$ atom sitting in the hcp site or in the fcc hollow (zinc blende phase), as shown in figure 15 , is $<0.05 \mathrm{eV}$, in agreement with DFT studies [39]. This explains how, using very little energy, some films have formed the zinc blende structure during growth.

During our simulations, we observed stacking faults and phase boundaries. Figure 16 illustrates a zinc blende phase within a wurtzite structure, which forms a phase boundary on the surface. $6 \mathrm{O}$ atoms sit in fcc hollows, in the Cc stack. Correct wurtzite sites are indicated and it is clear that a shift of the whole zinc blende phase to wurtzite would result in $4 \mathrm{O}$ 


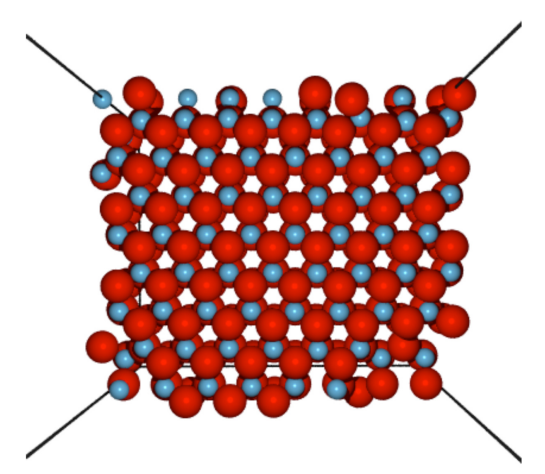

$\mathrm{AaBbAa}$

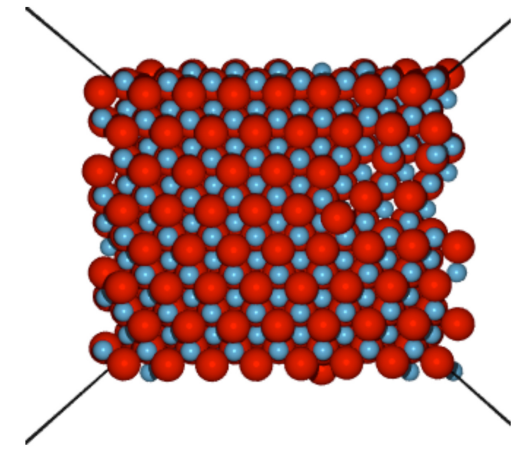

$\mathrm{AaBbCc}$

Figure 14: View from above, where three monolayers of $\mathrm{ZnO}$ have been sliced out in order to illustrate the stacking sequences. The image on the left shows the wurtzite structure with AaBbAa... stacking, and on the right is the zinc blende AaBbCc.. structure. In the wurtzite structure the fcc hollows are clearly seen throughout the film, whereas the zinc blende structure involves filling these fcc hollows. Smaller spheres represent Zn, whilst larger red spheres represent $\mathrm{O}$.

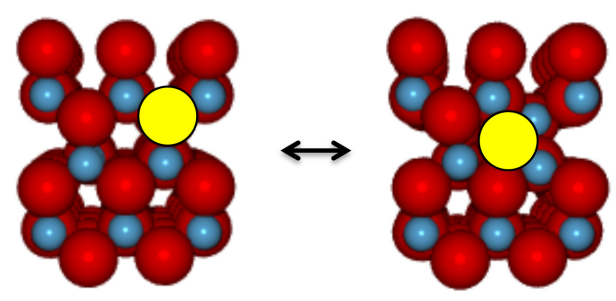

Figure 15: Growth switching between the preferred, more stable wurtzite structure and the zinc blende structure often occurs during a simulation. The energy difference for the $\mathrm{O}$ atom, marked in yellow, to switch to the zinc blende phase is $<0.05 \mathrm{eV}$. This small difference in energy enables atoms and layers to switch to the zinc blende phase.

vacancies, hence confirming an $\mathrm{O}$ deficiency in the film, which is common in $\mathrm{ZnO}$ deposited films [40,41]. This leads to a phase change, where zinc blende forms within the wurtzite phase with a phase boundary. The presence of a phase boundary in a film will change optical and electrical properties of the film. For such systems that are observed to be $\mathrm{O}$ deficient, we see a mixture of wurtzite and zinc blende phases in a single layer. The phase boundary between these two phases is $\mathrm{O}$ deficient. It is not clear whether it is the presence of the phase boundary that drives the $\mathrm{O}$ deficiency, or the presence of the $\mathrm{O}$ deficiency that drives the formation of a phase boundary.

Pre-annealed, evaporated $\mathrm{ZnO}$ films almost all exhibit stacking faults or a phase boundary. Sputtered films, however, do not all exhibit faults. The evaporation growth, shown in figure 2 , illustrates a phase boundary even when annealed at the lower temperature (figure 3 ). When evaporation deposition was simulated using an $\mathrm{O}$ deficient distribution of deposition species, the pre-annealed film had a mixture of wurtzite and zinc blende phases. Annealing this film 


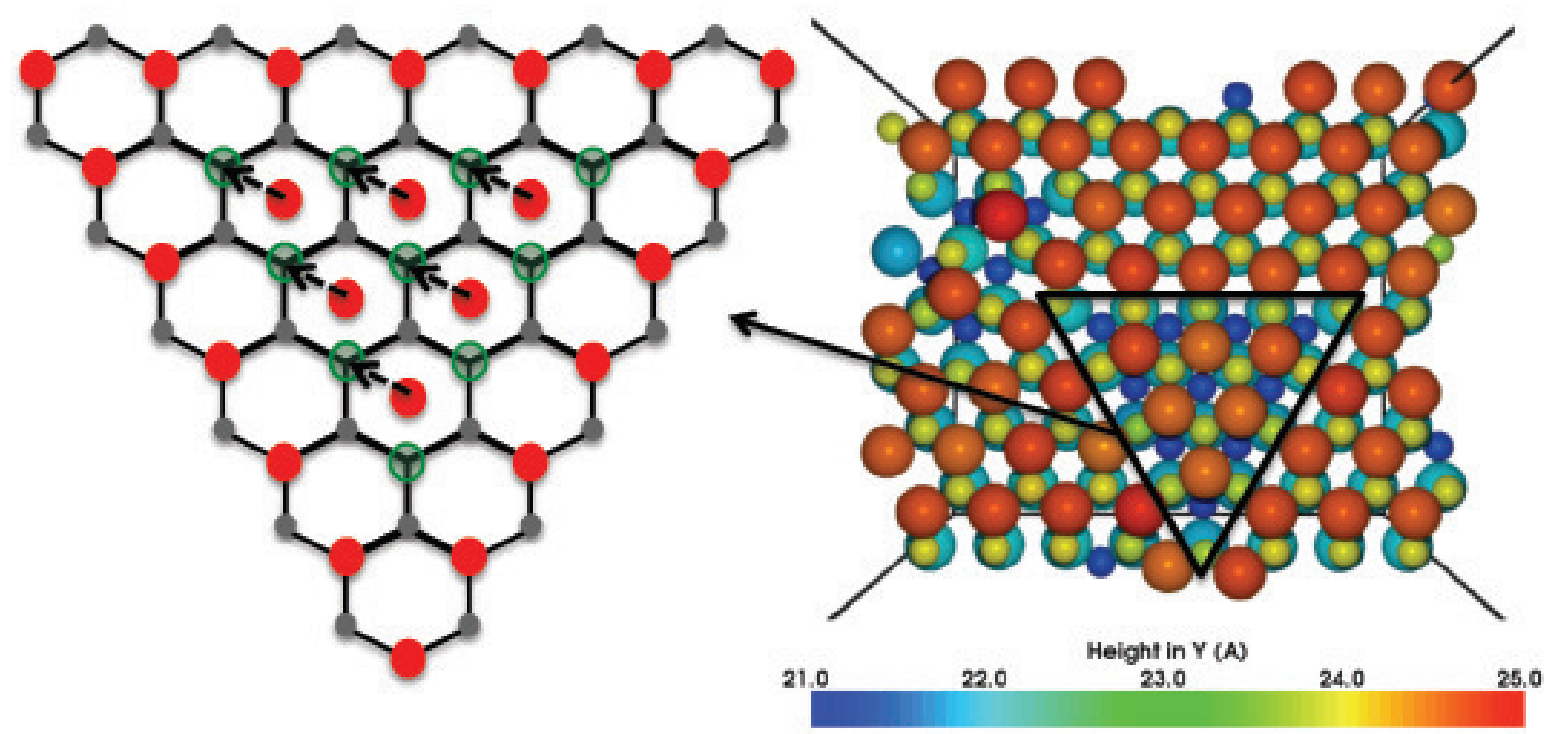

Figure 16: Sputtered growth using the $\mathrm{O}$ deficient distribution of deposition species. Annealing at $920 \mathrm{~K}$ attempts to recrystallise the film, however, due to an already inherent defect in the layer and an $\mathrm{O}$ deficit, annealing actually forms a phase boundary in the layer. On the right is an image taken from above a stacking fault. The highlighted area shows a zinc blende phase within a wurtzite structure, forming a phase boundary. On the left is a simple, atomistic diagram of the stacking fault where the light, transparent green circles represent an $\mathrm{O}$ vacancy. Arrows show clearly where atoms would need to diffuse for reordering to the wurtzite phase.

at $920 \mathrm{~K}$ actually changed the phase of the new layers to zinc blende. Once a film has inherent defects and stacking faults, annealing at temperature is not always sufficient to recrystallise and reorder the atoms into the wurtzite phase. As previously discussed, it costs $<0.05 \mathrm{eV}$ for $\mathrm{O}$ ad-atoms to shift from a wurtzite to zinc blende site, suggesting that during annealing, the heated atoms could cross this barrier to change phase.

All simulations of sputter deposition initially followed the correct AaBbAa wurtzite structure, at least in the first few new layers (only the first and second new layers are considered due to a steady state not yet being reached). The O deficient simulation, shown in figure 16, shows that annealing does not always have the ability to recrystallise $\mathrm{ZnO}$ into the wurtzite structure. If a defect or $\mathrm{O}$ deficiency is already present in the film then there is not always enough kinetic energy for the defects to stabilise and diffuse to correct sites.

\section{Conclusions}

Otf-KMC combined with traditional MD allows simulation of thin film growth over realistic time scales. In this paper, the simulations have modelled the equivalent of $\sim 1$ second of film growth, which using traditional MD would consume millions of computing years. The method has the ability to identify and analyse complicated growth mechanisms observed 
during growth on the atomistic scale. Understanding and recognising mechanisms, some of which are non-intuitive, concerted multi-atom motions, enables a more precise understanding of how thin film growth takes place.

$\mathrm{ZnO}$ film growth was simulated for $\sim 1$ second of real deposition time, allowing for the deposition of three monolayers of atoms onto the O-terminated wurtzite substrate. Different distributions of deposition species were used in order to simulate stoichiometric, $\mathrm{O}$ rich and $\mathrm{O}$ deficient environments. Stacking faults and phase boundaries have been observed during many of our simulations. During some simulations, part or all of a layer switches to the zinc blende phase requiring very little energy. Growth can switch to the zinc blende phase creating a stacking fault, or if only part of a layer has switched to the zinc blende phase, then this is a phase boundary. These phase boundaries have been found to occur more often in $\mathrm{O}$ deficient layers, however, it is unclear whether the phase boundary is caused by an O deficiency or vice versa.

Deposition by evaporation of stoichiometric, $\mathrm{O}$ rich and $\mathrm{O}$ deficient distributions of deposition species produced incomplete films with various vacancies, defects and inherent stacking faults. The best quality film resulted from purely $\mathrm{ZnO}$ units impacting upon the surface, where the film was almost stoichiometric (only $0.001 \% \mathrm{O}$ deficient). The film has an almost perfectly complete and crystalline first layer. However, the second layer exhibits a phase boundary whereby some of the layer is in the correct wurtzite phase and some of the layer has switched to the zinc blende phase.

To simulate the annealing treatment, the deposited film is heated up using MD. The films deposited by evaporation are annealed at $770 \mathrm{~K}$ and $920 \mathrm{~K}$. The lower temperature annealing produces a lattice which no longer has point defects in the first layer, but the second layer still exhibits a phase boundary. The higher temperature, however, promoted recrystallisation by eradicating phase boundaries and stacking faults.

Sputter deposition transfers more kinetic energy to the substrate from the higher energy impacts, hence growth is generally more complete, dense and crystalline than evaporation growth, also exhibiting less stacking faults. The best distribution of deposition species was found to be the $\mathrm{O}$ rich distribution. The film is, however, slightly $\mathrm{O}$ deficient, confirmed by a statistical analysis showing that $0.1-0.2 \%$ of $\mathrm{O}$ or $\mathrm{O}_{2}$ reflects off the surface during deposition, resulting in a $0.03 \% \mathrm{O}$ deficiency. No $\mathrm{Zn}$ is observed to leave the surface, suggesting that $\mathrm{Zn}$ binds to the surface with a high energy. The $\mathrm{O}$ deficit means that the wurtzite structure cannot form correctly due to $\mathrm{O}$ vacancies in the layer, thus enabling the zinc blende phase to occur, resulting in these phase boundaries. Annealing of this film at $920 \mathrm{~K}$ enables the completion and recrystallisation of layers. A layer in the pre-annealed film which had a phase boundary is rectified by the post-annealing treatment. The film becomes denser and more crystalline, with a phase boundary now only in the third new layer, although this layer is not so important as a steady state for that layer will not be reached during our simulations. 
During the sputtering simulations, two variations of a stoichiometric distribution of deposition species were compared. The first distribution, sputtering $\mathrm{A}$, deposited largely $\mathrm{ZnO}$ units which resulted in a film with $10 \% \mathrm{O}$ deficiency. The second distribution, sputtering $\mathrm{B}$, deposited largely single species, causing increased sputtering and reflection and thus a higher $\mathrm{O}$ deficiency (18\%). The higher $\mathrm{O}$ deficiency led to a higher defect density, thus enabling the formation of stacking faults and phase boundaries. An $\mathrm{O}$ deficiency within a layer was found to exist due to the presence of $\mathrm{O}$ vacancies and the formation of a phase boundary, where $\mathrm{O}$ atoms sit in both zinc blende and wurtzite sites.

Methods used enabled the in-depth analysis of growth mechanisms seen during growth, including complicated concerted, multi-atom motions which are not intuitive. An initial mechanism observed during early stages of growth was the $\mathrm{Zn}$ ad-atom transportation to correct lattice sites via bonding to $\mathrm{O}$ ad-atoms or $\mathrm{O}_{2}$ dimers. These 2 or 3 atom clusters require as little as $0.1 \mathrm{eV}$ to diffuse across the surface, transporting $\mathrm{Zn}$ atoms to the correct lattice sites. Single $\mathrm{Zn}$ atoms on the surface also have the ability to diffuse alone, with energy barriers of $\sim 0.2 \mathrm{eV}$. An important mechanism to deal with $\mathrm{Zn}$ interstitials below the surface involves a $\mathrm{Zn}$ interstitial diffusing to a correct lattice site in the surface with only $0.09 \mathrm{eV}$.

$\mathrm{Zn}_{x} \mathrm{O}_{y}$ strings on the surface have been found to be of great importance in the growth of $\mathrm{ZnO}$, acting as a nucleation site for growth. These are formed by complicated, concerted motions where $0.44 \mathrm{eV}$ is required for the transition. Once formed, these strings move with energy barriers of between $0.2-0.3 \mathrm{eV}$ until neighbouring atoms and strings then join to form a larger structure of strings. This process occurs after some tens of milliseconds, with hexagonal structures appearing hundreds of milliseconds later once enough atoms have been deposited, allowing the strings to join together and diffuse correctly into the wurtzite structure.

These simulations have allowed a more detailed understanding of the process of $\mathrm{ZnO}$ film growth and the important mechanisms involved, a lot of which are non-intuitive. It is clear from this work that the deposition process used does play a significant role on the resulting surface morphology and film quality and that annealing treatments can be very useful for recrystallisation, although not all defects and phase boundaries can be eradicated even from this high temperature annealing.

\section{Acknowledgments}

The authors would like to thank EPSRC and TSB for financial support and Loughborough University for the High Performance Computing time. One of the authors (S. Blackwell) thanks Loughborough University for a postgraduate studentship. This work was funded by EPSRC grant no. EP/C524322/1. 


\section{References}

[1] Z. L. Wang. Zinc oxide nanostructures: growth, properties and applications. Journal of Physics: Condensed Matter, 16:R829-R858, 2004.

[2] T. Minami. Transparent conducting oxide semiconductors for transparent electrodes. Semiconductor Science and Technology, 20(4):S35-S44, 2005.

[3] O. A. Fouad, A. A. Ismail, Z. I. Zanki, and R. M. Mohamed. Zinc oxide thinfilms prepared by thermalevaporation deposition and its photocatalytic activity. Applied Catalysis B: Environmental, 62(12):144-149, 2006.

[4] F. Chowdhury. Influence of thickness variation on the optical properties of $\mathrm{ZnO}$ thin films prepared by thermal evaporation method. Journal of Electron Devices, 10:448-455, 2011.

[5] F. C. M. van de Pol, F. R. Blom, and Th. J. A. Popma. R.F. planar magnetron sputtered ZnO films i: Structeral properties. Thin Solid Films, 204:349-364, 1991.

[6] U. Özgür, Y. I. Alivov, C. Liu, A. Teke, M. A. Reshchikov, S. Dogan, V. Avrutin, S. J. Cho, and H. Morkoçç. A comprehensive review of $\mathrm{ZnO}$ materials and devices. Journal of Applied Physics, 98:041301, 2005.

[7] D. Kohl, M. Luysberg, and M. Wuttig. Structural improvement of zinc oxide films produced by ion beam assisted reactive sputtering. Journal of Physics D: Applied Physics, 43:205301, 2012.

[8] S. Blackwell, R. Smith, S. D. Kenny, L. J. Vernon, and J. M. Walls. Modeling evaporation, ion-beam assist, and magnetron sputtering of $\mathrm{TiO}_{2}$ thin films over realistic timescales. Journal of Materials Research, 27(5):799-805, 2012.

[9] V. Georgieva, A. F. Voter, and A. Bogaerts. Understanding the surface diffusion processes during magnetron sputter-deposition of complex oxide Mg-Al-O thin films. Crystal Growth and Design, 11:2553-2558, 2011.

[10] G. Hu, G. Orkoulas, and P. D. Christofides. Model predictive control of film porosity in thin film deposition. In American Control Conference (ACC '09), pages 4797-4804, 2009.

[11] B. F. Gordiets, J. L. Andújar, C. Corbella, and E. Bertran. Kinetic model of thin film growth by vapor deposition. The European Physical Journal D - Atomic, Molecular, Optical and Plasma Physics, 35(3):505-511, 2005.

[12] S. Blackwell, R. Smith, and S. D. Kenny. Modeling evaporation, ion-beam assist, and magnetron sputtering of thin metal films over realistic time scales. Physical Review B, 86:035416-1-12, 2012.

[13] K. Y. Lee, C. Becker, M. Muske, F. Ruske, S. Gall, B. Rech, M. Berginski, and J. Hupkes. Temperature stability of ZnO:Al film properties for poly-Si thin-film devices. Applied Physics Letters, 91:241911, 2007.

[14] F. Ruske, M. Roczen, K. Lee, M. Wimmer, S. Gall, J. Hupkes, D. Hrunski, and B. Rech. Improved electrical transport in Al-doped zinc oxide by thermal treatment. Journal of Applied Physics, 107:013708, 2012.

[15] M. Warzecha, J. I. Owen, M. Wimmer, F. Ruske, J. Hotovy, and J. Hupkes. High mobility annealing of transparent conductive oxides. In E-MRS 2011 Fall Symposium I, volume 34 of IOP Conf. Series: Materials Science and Engineering, page 012004. IOP Publishing Ltd, 2012.

[16] M. Wimmer, F. Ruske, S. Scherf, and B. Rech. Improving the electrical and optical properties of DCsputtered ZnO:Al by thermal post deposition treatments. Thin Solid Films, 520(12):4203-4207, 2012.

[17] C. H. Bates, W. B. White, and R. Roy. New high-pressure polymorph of ZnO. Science, 137:993, 1962.

[18] K. Ellmer, A. Klein, and B. Rech. Transparent Conductive Zinc Oxide: Basics and Applications in Thin Film Solar Cells. Springer Series in Materials Science. Springer, 2008.

[19] Y. Ding and Z. L. Wang. Structures of planar defects in ZnO nanobelts and nanowires. Micron, 40(3):335342, 2009.

[20] Y. Ding, Z. L. Wang, T. Sun, and J. Qui. Zinc-blende $\mathrm{ZnO}$ and its role in nucleating wurtzite tetrapods and twinned nanowires. Applied Physics Letters, 90:153510, 2007.

[21] H. Morkoç and U. Özgür. Zinc Oxide: Fundamentals, Materials and Device Technology, chapter 1. General Properties of $\mathrm{ZnO}$, pages 1-76. Wiley-VCH, 2009.

[22] Y. Yan, M. M. Al-Jassim, K. M. Jones, S-H. Wei, and S. B. Zhang. Observation and first-principles calculation of buried wurtzite phases in zinc-blende CdTe thin films. Applied Physics Letters, 
Modelling the growth of ZnO thin films by PVD methods and the effects of post-annealing 20

77(10):1461-1463, 2000.

[23] Y. Yan, M. M. Al-Jassim, and K. M. Jones. Characterization of extended defects in polycrystalline CdTe thin films grown by close-space sublimation. Thin Solid Films, 389:75-77, 2001.

[24] Z. Ikonic, G. P. Srivastava, and J. C. Inkson. Optical properties of twinning superlattices in diamond-type and zinc-blende-type semiconductors. Physical Review B: Condsensed Matter, 52(19):14078-14085, 1995.

[25] A. F. Voter, F. Montalenti, and T. C. Germann. Extending the time scale in atomistic simulation of materials. Annual Review of Materials Research, 32:321-346, 2002.

[26] A. F. Voter. Radiation Effects in Solids, chapter Introduction to the Kinetic Monte Carlo Method. Springer, 2005.

[27] A. C. T. van Duin, S. Dasgupta, F. Lorant, and W. A. Goddard III. Reaxff: A reactive force field for hydrocarbons. Journal of Physical Chemistry A, 105:9396-9409, 2001.

[28] A. C. T. van Duin, A. Strachan, S. Stewman, Q. Zhang, X. Xu, and W. A. Goddard III. ReaxFF SiO reactive force field for silicon and silicon oxide systems. Journal of Physical Chemistry A, 107:3803-3811, 2003.

[29] D. Raymand, A. C. T. van Duin, M. Baudin, and K. Hermansson. A reactive force field (ReaxFF) for zinc oxide. Surface Science, 602(5):1020-1031, 2008.

[30] T. S. Herng, A. Kumar, C. S. Ong, Y. P. Feng, Y. H. Lu, K. Y. Zeng, and J. Ding. Investigation of the non-volatile resistance change in noncentrosymmetric compounds. Scientific reports, 2(587), 2012.

[31] H. J. C. Berendsen, J. P. M. Postma, W. F. van Gunsteren, A. DiNola, and J. R. Haak. Molecular dynamics with coupling to an external bath. Journal of Chemical Physics, 81(8):3684-3690, 1984.

[32] L. J. Vernon. Modelling the Growth of $\mathrm{TiO}_{2}$. Ph.D. thesis, Loughborough University, 2010.

[33] G. Henkelman, B. P. Uberuaga, and H. Jónsson. A climbing image nudged elastic band method for finding saddle points and minimum energy paths. Journal of Chemical Physics, 113(22):9901, 2000.

[34] G. Henkelman and H. Jónsson. Improved tangent estimate in the nudged elastic band method for finding minimum energy paths and saddle points. Journal of Chemical Physics, 113(22):9978, 2000.

[35] G. Henkelman, D. Sheppard, and R. Terrell. Optimization methods for finding minimum energy paths. Journal of Chemical Physics, 128(134106):1-10, 2008.

[36] G. H. Vineyard. Frequency factors and isotope effects in solid state rate processes. Journal of Physics and Chemisty of Solids, 3:121, 1957.

[37] T. David, S. Goldsmith, and R. L. Boxman. Dependence of zinc oxide thin film properties on filtered vacuum arc deposition parameters. Journal of Physics D: Applied Physics, 38(14):2407, 2005.

[38] S. W. Whangbo, H. K. Jang, S. G. Kim, M. H. Cho, K. Keong, and C. N. Whang. Properties of ZnO thin films grown at room temperature by using ionized cluster beam deposition. Journal of the Korean Physical Society, 37(4):456-460, 2000.

[39] B. Meyer. First-principles study of the polar O-terminated $\mathrm{ZnO}$ surface in thermodynamic equilibrium with oxygen and hydrogen. Publication: Physical Review B, 69(4):045416, 2004.

[40] E. S. Jung, J. Y. Lee, H. S. Kim, and N. W. Jang. Structural and optical characteristics of ZnO films with oxygen content. Journal of the Korean Physical Society, 47:S480-S484, 2005.

[41] C. Li, X. C. Li, P. X. Yan, E. M. Chong, Y. Liu, G. H. Yue, and X. Y. Fan. Research on the properties of $\mathrm{ZnO}$ thin films deposited by using filtered cathodic arc plasma technique on glass substrate under different flow rate of $\mathrm{O}_{2}$. Applied Surface Science, 253:4000-4005, 2007. 